\title{
Migration on a large scale
}

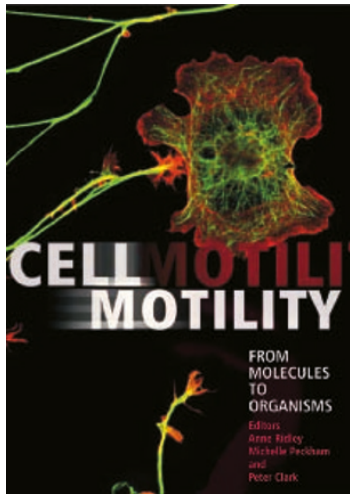

\section{Cell Motility: from Molecules to Organisms}

\author{
Edited by Anne Ridley, Michelle
}

Peckham and Peter Clark

John Wiley \& Sons • 2004

$\$ 180 / £ 90$

\section{Sandrine Etienne-Manneville}

Cell migration is a fundamental process in the developing and adult organism - under both normal and pathological conditions. Since the original description of migrating cultured cells by Michael Abercrombie in the early 1970s, the study of the molecular mechanisms underlying this complex cellular behaviour has garnered considerable attention. During the last decade, a comprehensive analysis of cell motility has resulted in much progress. The successful combination of genetics, biochemical and cell biology techniques has, in particular, led to the identification of many key factors involved in regulating the cytoskeleton. Because cell motility is involved in so many different cellular and developmental processes, covering the spectrum of topics from molecular regulation of cytoskeleton dynamics to control of cell migration during development is difficult to accomplish in a single book. Undeterred, however, Anne Ridley, Michelle Peckham and Peter Clark have undertaken this fantastic challenge as the editors of Cell Motility: From Molecules to Organisms, which is dedicated to Joan Heaysman, a long-time collaborator of Michael Abercrombie.

Contributed to by more than 50 researchers participating in the 5 th Abercrombie Symposium on Cell Motility (held in Oxford, UK, September 2003), this book consists of 20 chapters each of which can be read independently of the others. The references are, on the whole, remarkably up to date - an important consideration for a book covering this rapidly evolving area of research. The entire book would have gained from a more detailed preface containing an overview of the principles of cell migration and a more clearly defined organization of the chapters; however, each chapter generally provides an introduction that will allow readers unfamiliar with this field to place it within the context of cell migration.

Cell migration requires production of lamellipodia (membrane protrusions) at the leading edge. This volume begins with several chapters that summarize the enormous progress in understanding the molecular basis of this phenomenon. Actin-regulatory factors - such as the

Sandrine Etienne-Manneville is at CNRS-UMR144-Institut Curie, 26 rue d'Ulm, 75248 Paris, cedex 05, France.

e-mail: sandrine.etienne-manneville@curie.fr
Arp2/3 complex, cofilin, profilin, capping proteins, villin, and their regulators such as WASP, WAVE and VASP - form the basis of about one third of the book. These chapters give an excellent overview of how actin remodelling is regulated and its involvement in cell motility. As in most multi-authored books, there is some redundancy in content. For example, the regulation of the Arp2/3 complex is described in different contexts and degrees of detail in four chapters. A description of the molecules directly involved in actin dynamics is nicely complemented by two chapters on myosins that link actin dynamics to the plasma membrane, and the regulation of adhesion sites. A very interesting chapter on calpains describes the role of these proteases in governing cell adhesion. Emerging views on the contribution of microtubules to cell adhesion and cell motility are also highlighted. In coordination with cytoskeletal remodelling, cell migration requires a reorganization of membrane transport. This is elaborated on in detailed discussions on the contribution of ARF6 and dynamin — two unrelated GTPases that participate in the coordination of endocytic transport with cortical actin remodelling. Approximately two thirds of the book is devoted to the molecular basis of intrinsic cell motility, but it also touches on control of cell migration by external factors. Two chapters focus on the intracellular signals transduced during cell-cell interaction, and the contribution of ephrin receptors and cadherins to the migratory phenotype of neuronal growth cones and epithelial cells. Molecular mechanisms underlying chemotaxis are also summarized in additional chapters. Unfortunately, however, these chapters do not cover recent and significant findings that provide new molecular insights into chemotaxis; in particular, the response to the stimulation of G-protein-coupled receptors.

The progress of research on cell migration has been greatly aided by diverse in vitro cellular models. Chapter seven is singular in that it is dedicated to reviewing the most recent cell imaging techniques and recapitulating their principles and main applications. This chapter will be particularly useful for the novice in these technical approaches and should help them understand recent observations made with cell models. Finally, the last three chapters cover recent advances made in understanding cell migration in vivo during frog and mouse embryogenesis, and in situ in migrating neural and myogenic precursor cells. Unlike cells in culture, cells migrating in vivo encounter a mixture of soluble factors from the surrounding tissue, and move within a threedimensional matrix of extracellular proteins. The mechanisms of cell migration in this context are only just beginning to be addressed, and may be different from those involved in culture systems.

The emphasis of this book is resolutely on the molecular basis of motility mechanisms and my only serious complaint regards the scant coverage of other aspects of cell motility. Given the significance of cell migration in pathological situations, it would have been useful to include a specific chapter on cell invasion or metastasis, highlighting the deviation of normal migration, and the potential for targeting this process in pharmaceutical and clinical settings. Although some chapters may contain excessive detail and may assume too much prior knowledge for readers unfamiliar with cytoskeleton regulation, this book should prove to be an invaluable resource for graduate students and post-doctoral researchers interested in cell motility. 